\title{
ON $\mathcal{T}$-NONCOSINGULAR MODULES
}

\author{
DERYA KESKIN TÜTÜNCÜ® ${ }^{\bowtie}$ and RACHID TRIBAK
}

(Received 15 January 2009)

\begin{abstract}
In this paper we introduce $\mathcal{T}$-noncosingular modules. Rings for which all right modules are $\mathcal{T}$-noncosingular are shown to be precisely those for which every simple right module is injective. Moreover, for any ring $R$ we show that the right $R$-module $R$ is $\mathcal{T}$-noncosingular precisely when $R$ has zero Jacobson radical. We also study the $\mathcal{T}$-noncosingular condition in association with (strongly) FI-lifting modules.
\end{abstract}

2000 Mathematics subject classification: primary 16D10; secondary 16D80.

Keywords and phrases: $\mathcal{T}$-noncosingular module, (strongly) FI-lifting module.

\section{Introduction}

Throughout this paper $S$ denotes the endomorphism ring of any module $M$. In [8], the authors investigate $\mathcal{K}$-nonsingular modules. Motivated by this work, we introduce the notion of $\mathcal{T}$-noncosingular modules as the dual notion to the notion of $\mathcal{K}$-nonsingular modules. A module $M$ is called $\mathcal{T}$-noncosingular if, for every nonzero endomorphism $\varphi$ of $M, \operatorname{Im} \varphi$ is not small in $M$. Following [10], the module $M$ is called noncosingular if for every nonzero module $N$ and every nonzero homomorphism $f: M \rightarrow N, \operatorname{Im} f$ is not a small submodule of $N$. It is clear that every noncosingular module is $\mathcal{T}$-noncosingular.

The aim of this paper is to study $\mathcal{T}$-noncosingular modules. It turns out that some results about $\mathcal{K}$-nonsingular modules have corresponding duals for $\mathcal{T}$-noncosingular modules.

Section 2 introduces the concept of $\mathcal{T}$-noncosingular modules. The structure of finitely generated $\mathcal{T}$-noncosingular $\mathbb{Z}$-modules is described. We show that in general the direct sum of $\mathcal{T}$-noncosingular modules is not a $\mathcal{T}$-noncosingular module. Then we provide a necessary and sufficient condition for a direct sum of $\mathcal{T}$-noncosingular modules to be $\mathcal{T}$-noncosingular. We also prove that $\mathcal{T}$-noncosingularity is inherited by direct summands.

(C) 2009 Australian Mathematical Publishing Association Inc. 0004-9727/2009 \$16.00 
Section 3 is concerned with the concept of FI-lifting modules. We prove some results concerning these types of modules using the notion of $\mathcal{T}$-noncosingularity. In particular, any $\oplus$-supplemented module is FI-lifting.

\section{2. $\mathcal{T}$-noncosingular modules}

Let $M$ and $N$ be two modules. We say that $M$ is $\mathcal{T}$-noncosingular relative to $N$ if, for every nonzero homomorphism $\varphi: M \longrightarrow N, \operatorname{Im} \varphi$ is not small in $N$. If $M$ is $\mathcal{T}$-noncosingular relative to $M$, we say that $M$ is $\mathcal{T}$-noncosingular. The ring $R$ is said to be right $\mathcal{T}$-noncosingular if the right $R$-module $R_{R}$ is $\mathcal{T}$-noncosingular. Left $\mathcal{T}$-noncosingular rings are defined similarly.

Recall (see, for example, $[11,23.1]$ ) that a module $M$ is called cosemisimple if each factor module of $M$ has zero (Jacobson) radical and, for any ring $R$, the right $R$-module $R_{R}$ is cosemisimple precisely when every simple right $R$-module is injective, that is, $R$ is a right $V$-ring. Note, from the above definition, that every module with zero radical is $\mathcal{T}$-noncosingular. Consequently every cosemisimple module is $\mathcal{T}$-noncosingular.

It is clear that a module $M$ is noncosingular if and only if it is a $\mathcal{T}$-noncosingular module relative to $N$ for every module $N$. However, it is easy to check that the $\mathbb{Z}$-module $M=\mathbb{Z} / p \mathbb{Z}$, where $p$ is a prime integer, is $\mathcal{T}$-noncosingular but not noncosingular.

For every module $M$, let

$$
\bar{Z}(M)=\bigcap\{\operatorname{Ker} g \mid g: M \rightarrow T, \text { where } T \text { is small in its injective hull }\}
$$

and let $\nabla(M)=\{\varphi \in S \mid \operatorname{Im} \varphi \ll M\}$. It is easy to see that $\nabla(M)$ is an ideal of $S$. By the $\mathcal{T}$-noncosingular submodule of $M$ we mean $\bar{Z}_{\mathcal{T}}(M)=\bigcap_{\varphi \in \nabla(M)} \operatorname{Ker} \varphi$.

A module $M$ is called a lifting module if for every submodule $N$ of $M$, there is a decomposition $M=M_{1} \oplus M_{2}$ such that $M_{1} \leq N$ and $N \cap M_{2} \ll M_{2}$ or, equivalently, for every submodule $N$ of $M$ there is a direct summand $K$ of $M$ such that $N / K \ll$ $M / K$. The module $M$ is called discrete if it is lifting and satisfies the condition that, if $N$ is a submodule of $M$ for which $M / N$ is isomorphic to a direct summand of $M$, then $N$ is a direct summand of $M$.

EXAMPLE 2.1. Every injective module over a right hereditary $\operatorname{ring} R$ is $\mathcal{T}$-noncosingular. In fact, let $f$ be an endomorphism of $M$ such that $\operatorname{Im} f \ll M$. Since $R$ is a right hereditary ring and $\operatorname{Im} f \cong M / \operatorname{Ker} f, \operatorname{Im} f$ is injective. Thus, $\operatorname{Im} f$ is a direct summand of $M$. Therefore, $f=0$.

Proposition 2.2. Let $M$ be a module. We have:

(i) $\quad M$ is $\mathcal{T}$-noncosingular if and only if $\bar{Z}_{\mathcal{T}}(M)=M$;

(ii) $\bar{Z}_{\mathcal{T}}(M)$ is a fully invariant submodule of $M$; moreover, $\bar{Z}(M) \subseteq \bar{Z}_{\mathcal{T}}(M)$;

(iii) if $M=\bigoplus_{i \in I} M_{i}$, then $\bar{Z}_{\mathcal{T}}(M) \subseteq \bigoplus_{i \in I} \bar{Z}_{\mathcal{T}}\left(M_{i}\right)$. 
PROOF. (i) This is clear.

(ii) Let $\varphi \in S$ such that $\operatorname{Im} \varphi \ll M$ and let $f \in S$. We have $\operatorname{Im} \varphi f \subseteq \operatorname{Im} \varphi$, and hence $\operatorname{Im} \varphi f \ll M$. Therefore, $\bar{Z}_{\mathcal{T}}(M)$ is fully invariant.

The inclusion $\bar{Z}(M) \subseteq \bar{Z}_{\mathcal{T}}(M)$ is clear from the definitions.

(iii) Since $\bar{Z}_{\mathcal{T}}(M)$ is fully invariant in $M$, we have $\bar{Z}_{\mathcal{T}}(M)=\bigoplus_{i \in I}\left(\bar{Z}_{\mathcal{T}}(M) \cap M_{i}\right)$. It is sufficient to show that $\bar{Z}_{\mathcal{T}}(M) \cap M_{i} \subseteq \bar{Z}_{\mathcal{T}}\left(M_{i}\right)$ for all $i \in I$.

Let $x_{i} \in \bar{Z}_{\mathcal{T}}(M) \cap M_{i}$ for a fixed $i \in I$. Let $\varphi_{i} \in \operatorname{End}\left(M_{i}\right)$ such that $\operatorname{Im} \varphi_{i} \ll M_{i}$. Extending $\varphi_{i}$ to $\overline{\varphi_{i}}: M \longrightarrow M$ by $\overline{\varphi_{i}} \mid M_{j}=0$ for $i \neq j$, we have $\operatorname{Im} \overline{\varphi_{i}} \ll M$. Thus, $\overline{\varphi_{i}}\left(x_{i}\right)=\varphi_{i}\left(x_{i}\right)=0$. Therefore, $x_{i} \in \bar{Z}_{\mathcal{T}}\left(M_{i}\right)$.

Proposition 2.3. Let $M$ be a $\mathcal{T}$-noncosingular module and let $N$ be a direct summand of $M$. Then $N$ is $\mathcal{T}$-noncosingular.

Proof. Let $M=N \oplus N^{\prime}$. Let $\varphi: N \rightarrow N$ with $\operatorname{Im} \varphi \ll N$. Consider the homomorphism $\varphi \oplus 0_{N^{\prime}}: N \oplus N^{\prime} \rightarrow N \oplus N^{\prime}$ defined by $\varphi \oplus 0_{N^{\prime}}\left(n+n^{\prime}\right)=\varphi(n)$. Now $\varphi \oplus 0_{N^{\prime}}\left(N \oplus N^{\prime}\right)=\varphi(N) \ll M$. Since $M$ is $\mathcal{T}$-noncosingular, $\varphi \oplus 0_{N^{\prime}}=0$, and hence $\varphi=0$.

Note that the $\mathbb{Z}$-module $\mathbb{Z}$ is $\mathcal{T}$-noncosingular, but $S=\operatorname{End}(\mathbb{Z})$ is not von Neumann regular. However, the following two results show that there is some connection between the $\mathcal{T}$-noncosingular condition and regular endomorphism rings.

Proposition 2.4. If $M$ is a $\mathcal{T}$-noncosingular discrete module, then $S$ is von Neumann regular.

Proof. By [7, Theorem 5.4], $\nabla(M)=J(S)$ the Jacobson radical of $S$ and $S / J(S)$ is von Neumann regular. However, since $M$ is $\mathcal{T}$-noncosingular, $\nabla(M)=0$.

Proposition 2.5. If $M$ is a module such that $S$ is von Neumann regular, then $M$ is $\mathcal{T}$-noncosingular.

Proof. Let $f \in S$ such that $\operatorname{Im} f \ll M$. Since $S$ is von Neumann regular, there exists $g \in S$ such that $f g f=f$. This gives that $f g$ is an idempotent. Hence $\operatorname{Im} f g$ is a direct summand of $M$. But $\operatorname{Im} f g \leq \operatorname{Im} f$. Thus $\operatorname{Im} f g \ll M$. So $f g=0$, and hence $f=f g f=0$.

Proposition 2.6. Let $M=x R$ be a cyclic module such that Ann(x), the right annihilator of $x$, is an ideal of $R$. Then $M$ is a $\mathcal{T}$-noncosingular module if and only if $\operatorname{Rad}(M)=0$.

Proof. Suppose that $M$ is a $\mathcal{T}$-noncosingular module and $\operatorname{Rad}(M) \neq 0$. Therefore there exists $a \in R$ such that $x a \neq 0$ and $x a \in \operatorname{Rad}(M)$. Consider the endomorphism $f$ of $M$ defined by $f(x \alpha)=x a \alpha$ for every $\alpha \in R$. The map $f$ is well defined since $\operatorname{Ann}(x)$ is an ideal of $R$. Thus, $\operatorname{Im} f \leq \operatorname{Rad}(M)$ and $f \neq 0$. However, $\operatorname{Rad}(M) \ll M$. Then $M$ is not $\mathcal{T}$-noncosingular, a contradiction. The converse is clear.

The following two corollaries are now immediate. 
COROLlary 2.7. A ring $R$ is right (left) $\mathcal{T}$-noncosingular if and only if $\operatorname{Rad}(R)=0$.

COROLlary 2.8. Let $M$ be a local module over a commutative ring $R$. Then $M$ is a $\mathcal{T}$-noncosingular module if and only if $M$ is a simple module.

COROLLARY 2.9. Let $M$ be a finitely generated module over a commutative principal ideal domain $R$. Then $M$ is a $\mathcal{T}$-noncosingular module if and only if $\operatorname{Rad}(M)=0$.

PROOF. This follows from Propositions 2.3, 2.6 and [9, Corollary, p. 179].

PROposition 2.10. A finitely generated $\mathbb{Z}$-module $M$ is a $\mathcal{T}$-noncosingular module if and only if $M=\mathbb{Z}^{(n)} \oplus K$ for some $n \in \mathbb{N}$ and semisimple module $K$.

PROOF. It is well known that every finitely generated $\mathbb{Z}$-module is a finite direct sum of cyclic modules. Since every direct summand of a $\mathcal{T}$-noncosingular module is a $\mathcal{T}$-noncosingular module, the Chinese remainder theorem implies that every cyclic torsion $\mathbb{Z}$-module is a $\mathcal{T}$-noncosingular module if and only if it is semisimple by Corollary 2.8. The result follows. On the other hand, it is clear that if $K$ is semisimple, then $\mathbb{Z}^{(n)} \oplus K$ is $\mathcal{T}$-noncosingular because $\operatorname{Rad}\left(\mathbb{Z}^{(n)} \oplus K\right)=0$.

Proposition 2.11. Let $\left(M_{i}\right)_{i \in I}$ be a family of modules. Then $M=\bigoplus_{i \in I} M_{i}$ is $a$ $\mathcal{T}$-noncosingular module if and only if $M_{i}$ is a $\mathcal{T}$-noncosingular module relative to $M_{j}$ for all $i, j \in I$.

ProOf. $(\Rightarrow)$ Let $(i, j)$ be any pair in $I \times I$. Let $\varphi \in \operatorname{Hom}\left(M_{i}, M_{j}\right)$ such that $\operatorname{Im} \varphi \ll M_{j}$. Consider the homomorphism $f: M_{i} \oplus M_{j} \rightarrow M_{i} \oplus M_{j}$ defined by $f\left(x_{i}+x_{j}\right)=\varphi\left(x_{i}\right)$ with $x_{i} \in M_{i}$ and $x_{j} \in M_{j}$. Then $\operatorname{Im} f=\varphi\left(M_{i}\right) \ll M_{i} \oplus M_{j}$. However, $M_{i} \oplus M_{j}$ is a $\mathcal{T}$-noncosingular module by Proposition 2.3. Thus, $f=0$ and hence $\varphi=0$. This completes the proof.

$(\Leftarrow)$ Let $f$ be an endomorphism of $M$ such that $\operatorname{Im} f \ll M$. Consider the homomorphisms $\pi_{i}: M \rightarrow M_{i}$ (the projections) and $\phi_{i}: M_{i} \rightarrow M$ (the inclusion maps). Let $(i, j)$ be any pair in $I \times I$. Since $\operatorname{Im}\left(f \phi_{i}\right) \ll M$, we have $\operatorname{Im}\left(\pi_{j} f \phi_{i}\right) \ll M_{j}$. By hypothesis, $\pi_{j} f \phi_{i}=0$. Now, for all $x \in M$, we have $f(x)=$ $\sum_{i \in I} \sum_{j \in I} \pi_{j}\left[f\left(\phi_{i}\left(\pi_{i}(x)\right)\right)\right]$ (The sum is finite.) Thus, $f=0$. Consequently, $M$ is a $\mathcal{T}$-noncosingular module.

In general, a direct sum of $\mathcal{T}$-noncosingular modules is not a $\mathcal{T}$-noncosingular module, as the following example shows.

If $R$ is a Dedekind domain, then $R$ is said to be proper if $R$ is not a field.

If $R$ is a proper Dedekind domain, then for each nonzero prime ideal $P$ of $R$, $R\left(P^{\infty}\right)$ will denote the $P$-primary component of the torsion $R$-module $K / R$, where $K$ is the quotient field of $R$.

ExAmple 2.12. Let $R$ be a proper Dedekind domain. Let $P$ be any nonzero prime ideal of $R$. Consider the module $M=R\left(P^{\infty}\right) \oplus R / P$ and the endomorphism $f: M \longrightarrow M$ defined by $f(x+\bar{y})=c y$ with $x \in R\left(P^{\infty}\right), y \in R$ and $c$ is a nonzero element of $R\left(P^{\infty}\right)$ such that $c P=0$. It is clear that $\operatorname{Im} f=c R$ which is nonzero 
and small in $M$. So $M$ is not a $\mathcal{T}$-noncosingular module. In particular, for any prime integer $p$, the $\mathbb{Z}$-module $\mathbb{Z}\left(p^{\infty}\right) \oplus \mathbb{Z} / p \mathbb{Z}$ is not a $\mathcal{T}$-noncosingular $\mathbb{Z}$-module.

PROPOSITION 2.13. The following are equivalent for a ring $R$.

(i) Every right $R$-module is $\mathcal{T}$-noncosingular.

(ii) Every right $R$-module is noncosingular.

(iii) $R$ is a right $V$-ring, that is, every simple right $R$-module is injective.

ProOF. (i) $\Rightarrow$ (ii) Let $M$ and $N$ be two modules. Since $M \oplus N$ is $\mathcal{T}$-noncosingular, $M$ is $\mathcal{T}$-noncosingular relative to $N$ by Proposition 2.11. Therefore, $M$ is noncosingular. The implications (ii) $\Rightarrow$ (iii) and (iii) $\Rightarrow$ (i) follow from [10, Proposition 2.5].

Proposition 2.14. Let $M$ be a $\mathcal{T}$-noncosingular module. If $N \leq X, X / N \ll M / N$ and $N$ is a direct summand of $M$, then $N$ is unique.

Proof. Let $M$ be $\mathcal{T}$-noncosingular. Assume that $X / N_{i} \ll M / N_{i}$ with $M=N_{i} \oplus P_{i}$, $i=1,2$ and assume that $N_{1} \neq N_{2}$. Without loss of generality, suppose that $N_{1} \nsubseteq N_{2}$. Consider the projections $\pi_{N_{1}}: M \rightarrow N_{1}$ and $\pi_{P_{2}}: M \rightarrow P_{2}$. Then we have the nonzero homomorphism $\varphi=\pi_{P_{2}} \pi_{N_{1}}$. On the other hand, $\operatorname{Im} \varphi=\left(N_{1}+N_{2}\right) \cap P_{2} \subseteq$ $X \cap P_{2} \ll P_{2}$ implies that $\varphi=0$, a contradiction. Therefore, $N_{1}=N_{2}$.

Let $M$ be a module and $N \leq M$. The submodule $N$ is called coclosed if $N / K \ll M / K$ implies $N=K$ for every submodule $K$ of $M$ contained in $N$. Let $K \leq N \leq M$. If $K$ is coclosed in $M$ and $N / K \ll M / K$, then $K$ is called a coclosure of $N$ in $M$. The module $M$ is called a $U C C$ module if every submodule of $M$ has a unique coclosure in $M$ (see [3]).

COROLlARY 2.15. Every lifting $\mathcal{T}$-noncosingular module is UCC.

Proposition 2.16. Let $M$ be a $\mathcal{T}$-noncosingular module and $X$ fully invariant in $M$. Let $N \leq X$ such that $X / N \ll M / N$ and $N$ a direct summand of $M$. Then $N$ is (unique) fully invariant in $M$.

Proof. Let $P$ be a submodule of $M$ such that $M=N \oplus P$. Assume that $N$ is not fully invariant in $M$. Then there exist an endomorphism $\varphi$ of $M$ and $x \in N$ such that $\varphi(x) \notin N$. Let $\psi=\pi_{P} \varphi \pi_{N}: M \rightarrow P$, where $\pi_{N}: M \rightarrow N$ and $\pi_{P}: M \rightarrow P$ are the projections. Note that $\psi \neq 0(\varphi(x) \notin N)$ and $\operatorname{Im} \psi \subseteq X \cap P \ll M$. This contradicts the fact that $M$ is $\mathcal{T}$-noncosingular. Thus, $N$ is fully invariant in $M$.

COROLLARY 2.17. We have the following results.

(i) Let $M$ be a noncosingular module and $X \leq M$. Let $N \leq X$ such that $X / N \ll M / N$ and $N$ is a direct summand of $M$. Then $N$ is unique.

(ii) Let $M$ be a noncosingular module and $X$ a fully invariant submodule of $M$. Let $N \leq X$ such that $X / N \ll M / N$ and $N$ is a direct summand of $M$. Then $N$ is unique and fully invariant in $M$. 
Proof. Part (i) follows from Proposition 2.14 while part (ii) follows from Proposition 2.16.

\section{FI-lifting and strongly FI-lifting modules}

A module $M$ is called FI-lifting if for every fully invariant submodule $N$ of $M$, there is a decomposition $M=M_{1} \oplus M_{2}$ such that $M_{1} \leq N$ and $N \cap M_{2} \ll M_{2}$ or, equivalently, for every fully invariant submodule $N$ of $M$ there is a direct summand $K$ of $M$ such that $N / K \ll M / K$. The module $M$ is called strongly FI-lifting if, for every fully invariant submodule $N$ of $M$, there is a fully invariant direct summand $K$ of $M$ such that $N / K \ll M / K$. It is easy to prove that any direct summand of a strongly FI-lifting module is strongly FI-lifting.

Let $M$ be a module. If $N \leq M$, then $N$ is called a supplement submodule of $M$ if there exists a submodule $K$ of $M$ such that $M=N+K$ and $N \cap K \ll N$ (in this case we say that $N$ is a supplement of $K$ in $M$ ). If every submodule of $M$ has a (direct summand) supplement in $M$, then $M$ is called ( $\oplus$-)supplemented. If for every submodule $N$ of $M$ there exists a submodule $K$ of $M$ with $M=N+K$ and $N \cap K \ll M$, then $M$ is called weakly supplemented.

By [6, Theorem 3.4], any finite direct sum of FI-lifting modules is again FI-lifting. The following two examples show that this property is not true in general for infinite direct sums of FI-lifting modules. Let $R$ be a discrete valuation ring with maximal ideal $m$. Let $M=\bigoplus_{i=1}^{\infty} R / m^{i}$ or $M=R^{\mathbb{N}}$. By [12, Corollary 2, p. 48], $\operatorname{Rad}(M)$ does not have a supplement in $M$. Since $\operatorname{Rad}(M)$ is a fully invariant submodule of $M, M$ is not FI-lifting. On the other hand, it is clear that $R / m^{i}(i \geq 1)$ and $R$ are lifting modules.

Proposition 3.1. Let $M$ be a $\mathcal{T}$-noncosingular module. Then $M$ is FI-lifting if and only if $M$ is strongly FI-lifting.

Proof. Let $M$ be FI-lifting and $X$ a fully invariant submodule of $M$. Then there exists a direct summand $N$ of $M$ such that $X / N \ll M / N$. By Proposition 2.16, $N$ is fully invariant in $M$. Thus, $M$ is strongly FI-lifting. The converse is clear.

COROLlary 3.2. Let $M$ be a noncosingular module. Then $M$ is FI-lifting if and only if $M$ is strongly FI-lifting.

The following proof uses the concept of a left semicentral idempotent of a ring $S$ : this is an idempotent $e$ of $S$ for which exe $=x e$ for all $x \in S$.

LEMMA 3.3. If $K$ is a fully invariant submodule of $M$ having a coclosure $L$ which is a fully invariant direct summand of $M$, then $L$ is the unique direct summand coclosure of $K$.

ProOF. By [1, Lemma 1.9] and our hypothesis, there is a left semicentral idempotent $e \in S$ such that $L=e(M)$ and $K / e(M) \ll M / e(M)$. Let $c \in S$ be an idempotent such that $K / c(M) \ll M / c(M)$. Then $(1-c)(M) \cap K \ll(1-c)(M)$. Let us show that 
$L=c(M)$. Since $K$ is fully invariant in $M$, we have $(1-c)(K)=(1-c)(M) \cap K$. Thus, $(1-c)(K) \ll M$. Therefore, $e(1-c)(K) \ll e(M)$ and hence $e(1-c)(K) \ll$ $K$ since $e(M) \subseteq K$. So $e(1-c) e(K) \subseteq e(1-c)(K) \ll K$. Then, since $e$ is left semicentral, $(1-c) e(K)=e(1-c) e(K) \ll K$ and $(1-c) e$ is an idempotent of $S$. Therefore, $(1-c) e(K)=0$. Since $e(M)=e(K)$, we have $(1-c) e(M)=0$, and hence $e=c e$. It follows that $e(M) \subseteq c(M)$. Since $c(M) / e(M) \subseteq K / e(M) \ll$ $M / e(M)$, we obtain $c(M)=e(M)$. This completes the proof.

Proposition 3.4. If $M$ is a strongly FI-lifting module and $K$ is a fully invariant submodule of $M$, then there exists a unique (fully invariant) direct summand $L$ of $M$ such that $K / L \ll M / L$.

PROOF. This follows from Lemma 3.3.

PROPOSITION 3.5. Let $M$ be an FI-lifting module and $X$ a fully invariant submodule of $M$. If one of the following conditions is satisfied, then $M / X$ is strongly FI-lifting:

(i) $M / X$ is indecomposable;

(ii) $M / X$ is $\mathcal{T}$-noncosingular.

Proof. By [6, Proposition 3.3], $M / X$ is FI-lifting.

(i) Clearly, indecomposable FI-lifting modules are strongly FI-lifting.

(ii) This follows from Proposition 3.1.

PROpOSITION 3.6. Let $M$ be a lifting (respectively noncosingular weakly supplemented FI-lifting) module such that every small submodule is fully invariant. Then every factor module of $M$ is lifting (respectively strongly FI-lifting).

Proof. Let $X, Y$ be submodules of $M$ such that $M=X+Y$ and $X \cap Y \ll M$. Note that $M /(X \cap Y)=X /(X \cap Y) \oplus Y /(X \cap Y)$. By hypothesis, $X \cap Y$ is fully invariant in $M$. If $M$ is lifting, then $M /(X \cap Y)$ is lifting by [2, 22.2]. Since the lifting property is inherited by direct summands, $M / X$ is lifting. Now assume that $M$ is a noncosingular weakly supplemented FI-lifting module. Then the result follows from [6, Proposition 3.3], Corollary 3.2 and the fact that any direct summand of a strongly FI-lifting module is strongly FI-lifting.

PROPOSITION 3.7. Let $M$ be a module. The following are equivalent:

(i) $M$ is FI-lifting;

(ii) every fully invariant submodule of $M$ has a direct summand supplement;

(iii) for each fully invariant submodule $X$ of $M$, there is a coclosed submodule $K$ of $M$ and a direct summand supplement $L$ of $K$ such that $K \leq X, X / K \ll$ $M / K$ and every homomorphism $f: M \rightarrow M /(L \cap K)$ can be lifted to an endomorphism $g: M \rightarrow M$, that is, such that $g(m)+(L \cap K)=f(m)$ for all $m \in M$. 
ProOF. (i) $\Leftrightarrow$ (ii) Let $X$ be a fully invariant submodule of $M$. First assume that $M$ is FI-lifting. Then there exists a decomposition $M=M_{1} \oplus M_{2}$ such that $M_{1} \leq X$ and $M_{2} \cap X \ll M_{2}$. Then $M=X+M_{2}$ and $M_{2}$ is a direct summand supplement of $X$. Conversely, let $K$ be a direct summand supplement of $X$ in $M$. Then $M=K+X=K \oplus K^{\prime}$ and $K \cap X \ll K$ for some submodule $K^{\prime}$ of $M$. Consider the natural projection map $\phi: M \rightarrow K^{\prime}$. Since $X$ is fully invariant,

$$
\phi(X)=(X+K) \cap K^{\prime}=M \cap K^{\prime}=K^{\prime} \leq X .
$$

Thus, $M$ is FI-lifting.

(i) $\Rightarrow$ (iii) Let $X$ be a fully invariant submodule of $M$. Since $M$ is FI-lifting, there exists a decomposition $M=L \oplus K$ such that $K \leq X$ and $X / K \ll M / K$. Since $L \cap K=0$, clearly any homomorphism $f: M \rightarrow M /(L \cap K)$ lifts to a $g: M \rightarrow M$.

(iii) $\Rightarrow$ (i) Let $X$ be a fully invariant submodule of $M$. By (iii), there is a coclosed submodule $K$ of $M$ and a direct summand supplement $L$ of $K$ such that $K \leq X$ and $X / K \ll M / K$. Since $K$ is a supplement in $M$ by [4, Proposition 3], it follows from [5, Lemma 2.2] that $K$ is a direct summand of $M$. Thus, $M$ is FI-lifting.

Proposition 3.8. Let $M$ be a module. The following are equivalent:

(i) $M$ is strongly FI-lifting;

(ii) every fully invariant submodule of $M$ has a supplement $K$ which is a direct summand of $M$ with $M=K \oplus N$ for some fully invariant submodule $N$ of $M$.

PROOF. We completely follow the proof of Proposition 3.7((i) $\Leftrightarrow$ (ii)).

Proposition 3.9. Let $M$ be an FI-lifting module and let $U$ be a fully invariant submodule of $M$. Then $M / U$ is FI-lifting. If, moreover, $U$ is coclosed in $M$, then $U$ is also FI-lifting.

Proof. By [6, Proposition 3.3], $M / U$ is FI-lifting. Assume that $U$ is coclosed in $M$. Let $V$ be a fully invariant submodule of $U$. Then $V$ is fully invariant in $M$. So, there exist submodules $K$ and $K^{\prime}$ of $M$ such that $M=K \oplus K^{\prime}, K^{\prime} \leq V$ and $K \cap V \ll K$. Thus, $U=V+(U \cap K)$. Since $U$ is fully invariant in $M, U=(U \cap K) \oplus\left(U \cap K^{\prime}\right)$. Hence, $U \cap K$ is a direct summand of $U$. Moreover, $V \cap(U \cap K)=V \cap K \ll K$. This implies that $V \cap(U \cap K) \ll U \cap K$ since $U \cap K$ is coclosed in $M$ by [2, 3.7]. Therefore, $U \cap K$ is a direct summand supplement of $V$ in $U$. By Proposition 3.7, $U$ is FI-lifting.

A module $M$ is called a duo module provided that every submodule of $M$ is fully invariant.

Proposition 3.10. Let $M$ be a module. Consider the following statements:

(i) $M$ is lifting;

(ii) $M$ is $\oplus$-supplemented;

(iii) $M$ is FI-lifting.

Then (i) $\Rightarrow$ (ii) $\Rightarrow$ (iii). If $M$ is a duo module, then (iii) $\Rightarrow$ (i). 
ProOF. (i) $\Rightarrow$ (ii) This is clear.

(ii) $\Rightarrow$ (iii) This is clear by Proposition 3.7.

The rest is clear from the definitions.

REMARK. (1) Consider the $\mathbb{Z}$-module $M=\mathbb{Z} / p \mathbb{Z} \oplus \mathbb{Z} / p^{3} \mathbb{Z}$. It is well known that $M$ is not lifting, but it is FI-lifting by [6, Theorem 3.4].

(2) Consider $\mathbb{Q}$ the additive group of rational numbers. Let $f$ be any nonzero $\mathbb{Z}$-endomorphism of $\mathbb{Q}$. Let $r$ be a nonzero element of $\mathbb{Q}$ such that $f(1)=r$. Let $a$ and $b$ be two nonzero integers. Then $f(1)=f((1 / b) \times b)=f(1 / b) b=r$. So $f(1 / b)=r / b$. Thus, $f(a / b)=f(1 / b) a=(r / b) a=(a / b) r$. Now let $N$ be a nonzero fully invariant submodule of $\mathbb{Q}$. Let $s$ be a nonzero element of $N$. Let $g$ be the endomorphism of $\mathbb{Q}$ defined by $g(x)=(1 / s) x$ for every $x \in \mathbb{Q}$. Since $N$ is fully invariant, $g(s) \in N$. Thus, $1 \in N$. Hence, $\mathbb{Q} \leq N$ since $h(1) \in N$ for every $h \in \operatorname{End}_{\mathbb{Z}}(\mathbb{Q})$. Consequently, the only fully invariant submodules of $\mathbb{Q}$ are 0 and $\mathbb{Q}$. Therefore, $\mathbb{Q}$ is strongly FI-lifting. On the other hand, $\mathbb{Q}$ is not $\oplus$-supplemented since $\mathbb{Q}$ is an indecomposable $\mathbb{Z}$-module which is not hollow.

THEOREM 3.11. Let $M$ be a $\mathcal{T}$-noncosingular module and $X$ a fully invariant submodule of $M$. Then $M$ is (strongly) FI-lifting if and only if $M=M_{1} \oplus M_{2}$ such that $M_{1}$ and $M_{2}$ are (strongly) FI-lifting and $M_{1}$ is the unique fully invariant direct summand of $M$ with $M_{1} \subseteq X$ and $X / M_{1} \ll M / M_{1}$.

Proof. $(\Rightarrow)$ Since $X$ is fully invariant in $M$ and $M$ is FI-lifting, there exists a decomposition $M=M_{1} \oplus M_{2}$ such that $M_{1} \subseteq X$ and $X / M_{1} \ll M / M_{1}$. By Proposition 2.16, $M_{1}$ is unique and fully invariant in $M$. Then by Proposition 3.9, $M_{1}$ and $M_{2}$ are FI-lifting. The remainder of the proof is a consequence of Propositions 2.3 and 3.1 .

$(\Leftarrow)$ This follows from [6, Theorem 3.4] and Proposition 3.1.

Proposition 3.12. Let $M=M_{1} \oplus M_{2}$. Then $M_{2}$ is FI-lifting if and only iffor every fully invariant submodule $N / M_{1}$ of $M / M_{1}$, there exists a direct summand $K$ of $M$ such that $K \leq M_{2}, M=K+N$ and $N \cap K \ll M$.

Proof. Suppose that $M_{2}$ is FI-lifting. Let $N / M_{1}$ be any fully invariant submodule of $M / M_{1}$. It is easy to see that $N \cap M_{2}$ is fully invariant in $M_{2}$. Since $M_{2}$ is FI-lifting, there exists a decomposition $M_{2}=K \oplus K^{\prime}$ such that $M_{2}=\left(N \cap M_{2}\right)+K$ and $N \cap K \ll K$. Clearly, $M=N+K$.

Conversely, suppose that $M / M_{1}$ has the stated property. Let $H$ be a fully invariant submodule of $M_{2}$. It is easy to see that $\left(H \oplus M_{1}\right) / M_{1}$ is fully invariant in $M / M_{1}$. By hypothesis, there exists a direct summand $L$ of $M$ such that $L \leq M_{2}, M=L+H+M_{1}$ and $L \cap\left(H+M_{1}\right) \ll M$. By modularity, $M_{2}=L+H$. It follows easily that $L$ is a supplement of $H$ in $M_{2}$. Therefore, $M_{2}$ is FI-lifting by Proposition 3.7 . 


\section{Acknowledgement}

This paper was written during a visit by the second author to the Department of Mathematics, Hacettepe University, and he thanks the first author and her family for their hospitality.

\section{References}

[1] G. F. Birkenmeier, B. J. Müller and S. T. Rizvi, 'Modules in which every fully invariant submodule is essential in a direct summand', Comm. Algebra 30(3) (2002), 1395-1415.

[2] J. Clark, C. Lomp, N. Vanaja and R. Wisbauer, 'Lifting modules', in: Supplements and Projectivity in Module Theory, Frontiers in Mathematics (Birkhäuser, Basel, 2006).

[3] L. Ganesan and N. Vanaja, 'Modules for which every submodule has a unique coclosure', Comm. Algebra 30(5) (2002), 2355-2377.

[4] T. Inoue, 'Sum of hollow modules', Osaka J. Math. 20 (1983), 331-336.

[5] D. Keskin, 'Discrete and quasi-discrete modules', Comm. Algebra 30(11) (2002), 5273-5282.

[6] T. Kosan, 'The lifting condition and fully invariant submodules', East-West J. Math. 7(1) (2005), 99-106.

[7] S. H. Mohamed and B. J. Müller, Continuous and Discrete Modules, London Mathematical Society Lecture Note Series, 147 (Cambridge University Press, Cambridge, 1990).

[8] S. T. Rizvi and C. S. Roman, 'On $\mathcal{K}$-nonsingular modules and applications', Comm. Algebra 35 (2007), 1-22.

[9] D. W. Sharpe and P. Vamos, Injective Modules (Cambridge University Press, Cambridge, 1972).

[10] Y. Talebi and N. Vanaja, 'Torsion theory cogenerated by $M$-small modules', Comm. Algebra 30(3) (2002), 1449-1460.

[11] R. Wisbauer, Foundations of Module and Ring Theory (Gordon and Breach, Philadelphia, PA, 1991).

[12] H. Zöschinger, 'Komplementierte Moduln über Dedekindringen', J. Algebra 29 (1974), 42-56.

DERYA KESKIN TÜTÜNCÜ, Department of Mathematics, Hacettepe University,

06800 Beytepe, Ankara, Turkey

e-mail: keskin@ hacettepe.edu.tr

RACHID TRIBAK, Département de Mathématiques, Faculté des Sciences de Tétouan, B.P. 21.21, Tétouan, Morocco e-mail: tribak12@yahoo.com 